\title{
ENTRE TIEMPO Y ETERNIDAD: LA IDEA DE LA EVITERNIDAD EN EL PENSAMIENTO DE RAIMUNDO LULIO
}

\author{
Klaus Reinhardt \\ Trier (Alemania)
}

\section{RESUMEN}

El objeto de este artículo lo constituye la idea luliana de la eviternidad que media entre la eternidad de Dios y la temporalidad del hombre. En el primer apartado se demuestra la presencia del término en las obras de Raimundo Lulio, de modo particular en su gran enciclopedia Arbor scientiae. Tras una breve exposición de la pre-historia de la idea luliana de la «eviternidad» o del «Evo» en el apartado segundo, en el tercero se destaca que Lulio, a diferencia de los demás teólogos medievales, no refiere la eviternidad al tiempo de los ángeles, sino que, para él, eviternidad es un concepto escatológico que significa la medida de duración de la vida de los salvados o condenados. El artículo concluye con un repaso a una controversia teológica actual en la que se discute la utilidad del término «evo» para aclarar el carácter de la vida eterna.

Palabras clave: Raimundo Lulio, eternidad, eviternidad, evo, tiempo.

\section{ABSTRACT}

The subject of this article is Lull's concept of eviernity which mediates between the eternity of God and the temporality of man. The first paragraph points out the salient importance of this topic in the work of Ramon Lull, particulary in his outstanding enciclopedia Arbor scientiae. After a glance backward at the previous history of the Lullian idea (second paragraph), the third paragraph sets forth that with his notion of eviternity Lull does not associate the special time of angels; unlike other medieval theologians for him eviternity is an escathological concept which signifies the measurement of the duration of a life in damnation or salvation. The article concludes with a view at a contemporary theological dispute about the usability of «aevum» as a term of the doctrine of eternal life.

Key words: Raimundo Luliò, eternity, eviternity, aevum, time.

Entre los artículos de fe que Raimundo Lulio pretende demostrar por razones necesarias ante infieles y fieles, figuran en primer lugar los de la Trinidad y de la Encarnación. ${ }^{1}$ Pero casi con el mismo afán, Lulio se dedica también a temas escatológicos y particularmente al tema de la vida eterna en el

1 Cf. sobre Raimundo Lulio en general, L. Badia - A. Bonner, Ramón Llull: vida, pensamiento y obra literaria, Madrid 1993. Una lista de las obras lulianas se encuentra en: A. Bonner (ed.), Selected Works of Ramon Llull, 2 vols., Princeton (N.J.) 1985, pp. 1257 ssq. y en M. Cruz Hernández, El pensamiento de Ramón Lull, Madrid 1977, pp. 361 ss. 
paraíso o el infierno. En este contexto surge su idea, a primera vista un poco extraña, de la eviternidad: los fieles deben esperar, según Lulio, no precisamente una vida eterna, sino una vida eviterna.

\section{PRESENCIA DEL TÉRMINO «EVITERNIDAD» EN LAS OBRAS DE RAIMUNDO}

En ninguna de sus obras Raimundo Lulio destaca la importancia del término «eviternidad» tanto cuanto en la gran enciclopedia Arbor scientiae, compuesta en Roma en los años 1295/96. Bajo el símbolo del árbol presenta una introducción enciclopédica a todos los saberes del hombre o, mejor dicho, un arte para hallar todas las verdades del mundo. ${ }^{2}$ Entre los 16 árboles en los que se multiplica el árbol general, el «arbor aeviternalis» ocupa el puesto número once. Le preceden los árboles elemental, vegetal, sensual, imaginal, humanal, moral, imperial (es decir, estatal), apostólica (es decir, eclesial), celestial (éste se refiere al cielo empíreo) y angelical. El árbol eviternal representa, pues, el último grado que puede alcanzar una criatura, porque significa la última perfección de los ángeles y de los hombres. Más allá del árbol eviternal siguen sólo el árbol maternal (de la Virgen Madre María), el árbol cristianal o divino-humanal y, como apogeo, el árbol divinal. Al final, Lulio pone dos árboles más, el árbol ejemplifical y el árbol cuestional, pero estos dos árboles no añaden nada a la pirámide del universo, sino que complementan los catorce árboles anteriores bajo un punto de vista más bien metodológico.

A primera vista, la ubicación del árbol eviternal encima del angelical y celestial no cuadra bien con el esquema del cosmos jerárquicamente ordenado. Pero hay que tener en cuenta que Lulio, bajo el símbolo del árbol, no pretende presentar una visión estática, sino más bien una evolución dinámica de un universo que se acerca cada vez más a su creador tri-uno. En este movimiento, el árbol eviternal no ocupa ciertamente el puesto supremo, pero sí el puesto más alto alcanzable por los ángeles y hombres en general, y que es superado sólo por la Virgen y por Jesucristo.

Esta visión dinámica, escatológica del hombre y del mundo aparece ya en uno de los primeros escritos de Lulio, en el Liber contemplationis (1274). ${ }^{3}$ En esta voluminosa obra sobre la con-

2 Edición del texto latino: Barcelona ca. 1489. Utilizo en este artículo la edición publicada en Lyon en 1637 (ejemplar en Madrid BN, R. 34610). Durante el congreso «Arbor scientiae. Der Baum des Wissens von Ramon Llull», del 29. 9 al 2.10.1997, organizada por el Raimundus-Lullus-Institut der Universität Freiburg en la misma ciudad, tuve la oportunidad de consultar el texto provisional de la nueva edición crítica del Arbor scientiae, preparada por Pere Villaiba (Barcelona); la edición va a salir pronto en la serie Corpus christianorum. Continuatio mediaevalis (CCCM) de la editorial Brepols, dentro de la colección Raimundi Opera Latina (ROL). Traducción española: Vida, arte magna y árbol de la ciencia de [...] Raymundo Lulio, traduzido y explicado por Alonso Cepeda y Adrada, Mallorca 1604; Bruselas 1663 (ejemplar en Madrid BN R. 17978). Sobre la simbología del árbol, tan usual en Ramón Llull, véase M. Cruz Hernández, «El símbolo del árbol en Ramón Llull e Ibn al-Jatib», en Studia lullistica. Miscellanea in honorem Sebastiani Garcias Palou, Mallorca 1989, pp. 19-24; D. Urvoy, «Le symbole de l'arbre chez les auteurs arabes antérieurs à Lull», en F. Domínguez - J. de Salas, Constantes y fragmentos del pensamiento luliano, Tübingen 1996, pp. 91-97. Sobre el contenido del Arbor scientiae, cf. Robert D. F. Pring-Mill, «El Microcosmos Iul.lià», en id̉., Estudis sobre Ramon Llull (19561978). A cura de Lola Badia i Albert Soler, Barcelona, 1991, especialmente pp. 55-56; M. Cruz Hernández, El pensamiento, o. c. en nota 1, pp. 125-44; T. y J. Carreras y Artau, Historia de la filosofía española. Filosofía cristiana de los siglos XIII al XV. Tomo I, Madrid 1939, pp. 403-422.

3 Edición del Magnus liber contemplationis in Deum en Beati Raymundi Lulli Opera, ed. I. Salzinger, tom. IX-X, Mainz 1740 y 1742; reimpresión: Frankfurt am Main 1965. La edición moguntina se cita a continuación bajo la sigla MOG. 
templación de Dios, Lulio expone cómo el hombre fue creado por Dios para alabarlo y amarlo «in aeternum». El abismo que media entre la eternidad de Dios y la caducidad del hombre es superado por Dios, ya que resucita al hombre a una vida inmortal y eterna.

No cabe duda de que el tema de la inmortalidad era para Lulio un tema existencial. Esto lo demuestra en el diálogo llamado Libro del gentil y de los tres sabios, ${ }^{4}$ compuesto hacia 1276 , en el que aparece, además, por primera vez (en cuanto yo sepa) el término «eviternidad».

El motivo para este diálogo lo constituye, como se dice en el prólogo, el temor del gentil, del filósofo pagano, a la muerte. La certeza de su muerte le llena de tristeza y desesperación, ya que no cree en Dios ni en la resurrección de los muertos. Al buscar a alguien que podría sanarle, el pagano encuentra en un bosque a tres sabios, un judío, un cristiano y un musulmán, que discuten sobre la verdad de sus religiones diferentes. A ellos se les apareció poco antes una dama noble, llamada inteligencia, quien les enseñó un nuevo modo de hallar la verdad, utilizando los árboles que rodeaban el sitio del encuentro como ejemplos y símbolos. El nuevo método no era sino el arte luliano, por el cual Raimundo Lulio pretende demostrar las verdades de la fe cristiana, partiendo de las dignidades, virtudes o principios absolutos de Dios (bonitas, magnitudo, aeternitas, potestas, sapientia, amor, perfectio) y argumentando no mediante autoridades, sino mediante razones inteligibles. Los tres sabios no tardan en aplicar las reglas de este arte para convencer al filósofo pagano de la existencia de Dios y de la resurrección de los muertos. He aquí algunos argumentos en pro de la resurrección de los muertos. Uno de los argumentos procede del siguiente modo: Porque la eternidad de Dios es idéntica con su bondad, y porque, además, un bien que dura sin fin es mejor que un bien que perece, hay que suponer que Dios resucita el cuerpo del hombre y lo hace durar eternamente. ${ }^{5}$ Otro argumento parte de la identidad de la eternidad divina con la sabiduría. ${ }^{6}$ Porque Dios demuestra su sabiduría principalmente mediante su justicia, hay que suponer la inmortalidad del hombre; si no, la justicia divina no podría premiar muchos méritos de los hombres ni imponerles la pena justa. En un tercer argumento, el punto de partida es la concordancia entre eternidad divina y amor humano de Dios: ${ }^{7}$ el hombre ama a Dios porque Dios es eterno. Si Dios resucita al hombre, éste puede amar a Dios mucho más que en el caso contrario. Entonces etc.

No entro a valorar en este contexto la argumentación luliana. Quiero llamar la atención, más bien, sobre el hecho de que Lulio no utiliza nunca en el primer libro del diálogo, al que se refieren los argumentos antes relatados, el término «eviternidad», sino que habla de un «esse seu durare in aeternum» o «in perpetuum» del hombre. Sólo en la segunda parte, en la que se expone la fe judía, introduce el término «eviternidad». El judío habla de «una eternidad (aeternitas) que se llama en latín evo (aevum), y es una eternidad (aeternitas) que tiene principio, pero no fin; está creada por la eternidad suprema, la cual es sin principio ni fin»; y añade: «la cual eviterni-

4 El libro fue compuesto en catalán y traducido más tarde al latín. Ediciones del texto catalán (Llibre del gentil e dels tres savis): ed. por Rosselló y M. Obrador en Obras de Ramon Lull, tom. 1, Palma de Mallorca 1901; ed. por S. Garcías Palou en Obres essencials, tom. 1, Barcelona 1957; ed. por A. Bonner en Nova edició de les obres de Ramon Llull, tom. 2, Palma de Mallorca, 1993. Edición de la traducción latina en MOG II, 21-114. Cf. Ramon Lull, Das Buch vom Heiden und den drei Weisen. Übersetzt und herausgegeben von Theodor Pindl, Stuttgart, 1998.

5 Liber de gentili et tribus sapientibus, lib. 1, arbor 1.

6 Ibíd.

7 Ibíd. o. c., lib. 1, arbor 2. 
dad (aeviternitas) la ha creado Dios para premiar a los hombres por su humildad y para castigarlos por su soberbia». ${ }^{8}$ Este enunciado está situado en un contexto en el que el judío combate la tesis (arrogante, a sus ojos) de un mundo eterno y defiende la fe humilde en la creación del mundo y del hombre por parte de Dios.

De lo anteriormente expuesto uno podría deducir que Raimundo Lulio había encontrado el término «eviternidad» en pensadores judíos. Pero hasta ahora no se conocen textos que confirmen esta hipótesis. En todo caso, en sus escritos posteriores Lulio se quedó, en un primer momento, con el modo de hablar de la vida eterna o perpetua del hombre, practicado en la primera parte del Libro del gentil y de los tres sabios, sin utilizar el término «eviternidad». ${ }^{9}$ Sólo a partir del año 1294, el termino de la eviternidad vuelve a aparecer en los escritos lulianos, por ejemplo en la Lectura super Artem inventivam et Tabulam generalem, ${ }^{10}$ compuesta en los años 1294/95 en Roma y Nápoles, y poco más tarde en el ya citado Arbor scientiae. En los escritos ulteriores se encuentra el término con mucha frecuencia. ${ }^{11}$

\section{PREHISTORIA DE LA DISTINCIÓN LULIANA ENTRE ETERNIDAD Y EVITERNIDAD}

Santo Tomás de Aquino presenta la distinción entre evo y eternidad en su comentario al Liber de causis, diciendo que es una distinción nueva y, además, arbitraria, porque los dos términos se diferencian sólo como los términos anthropos y homo, es decir, como la palabra griega y su equivalente latino. ${ }^{12}$ Efectivamente, los términos «aevum», «aevitas», «aetas», «aeternitas» y «aeviternitas» remontan todos a la palabra griega « $\alpha \iota \omega v »$, reproducida en latín como «aevum». Del término «aevum» derivan el adjectivo «aeviternum» y los substantivos «aevitas» y «aeviternitas», que se convierten por contracción en «aetas», «aeternum» y «aeternitas». «Aion/aevum» puede signi-

8 Raimundus Lullus, Liber de gentili et tribus sapientibus, lib. II, art. 2 (De creatione), apartado De aeternitate et superbia: «Judaeus autem respondit: hoc per quod divina aeternitas in homine convenit cum humilitate contra superbiam, est per influentiam doni divinae gratiae quae datur homini contra culpam superbiae. Et ex hoc significatur una aeternitas, quae latine aevum dicitur, et est aeternitas quae habet principium, sed non finem; et est creata a suprema Aeternitate quae est sine principio et fine; quam aeviternitatem Deus creavit ad remunerandum homines propter humilitatem et ad puniendum homines propter superbiam, ut homo habeat cognitionem florum primae Arboris aeternaliter sine fine». MOG II, $1722,26(46)$.

9 Cf. Liber de quatuordecim articulis fidei, dist. 5: De glorificatione, en: MOG II, 159 («quia habet potestatem conservandi eum in perpetuo esse»); Quaestiones per artem demonstrativam seu inventivam solubiles, en MOG IV, 54-64 (De altera vita. q. 41: «Utrum alia vita sit in aeternum»); Ars inventiva veritatis, en MOG V, 111-121 («Utrum sit alia vita perpetua hominibus»).

10 Lectura super Artem inventivam et Tabulam generalem dist. 3, pars 1, q. IX: De paradiso, q. 7: De tempore paradisi, en: MOG V, pp. 285-288 (643-646)

11 Esto se puede comprobar fácilmente mediante el CD-ROM que contiene las obras del último período de Lulio, publicadas en la serie Raimundi Lulli Opera Latina en el Corpus christianorum. Continutio mediaevalis. El término «eviternidad» o sus derivados aparecen allí 102 veces.

12 Thomas de Aquino, «Expositio super librum de causis, lectio 2 », en id. Opuscula omnia, ed. P. Mandonnet, Paris 1927, tom. I, 202: «Sed si quis recte consideret, aevum et aeternitas non differunt nisi sicut anthropos et homo: in graeco enim aevum aeternitas dicitur, et anthropos homo». 
ficar tanto un período largo (por ejemplo, el medio evo) como una duración ilimitada y, por consiguiente, eterna en el sentido estricto de la palabra. ${ }^{13}$

En la edad antigua no encontramos todavía una distinción estricta entre evo y eternidad. ${ }^{14}$ Pero sí se notan diferencias en el uso de las dos palabras. Los filósofos prefieren el término eternidad y lo entienden en el sentido de eternidad ilimitada. En el lenguaje poético se usa más la palabra evo en el doble sentido de duración larga o de duración ilimitada. Así, por ejemplo, Boecio en su obra Philosophiae consolatio utiliza en la poesía la palabra «aevum» en el sentido de eternidad: «O qui perpetua mundum ratione gubernas /terrarum caelique sator, qui tempus ab aevo/ire iubes $[\ldots] »,{ }^{15}$ mientras que en la prosa filosófica prefiere el término «aeternitas», como lo demuestra su bien conocida definición: «Aeternitas igitur est interminabilis vitae tota simul et perfecta possessio". ${ }^{16}$ Boecio, pues, no distingue exactamente entre evo y eternidad, pero reconoce otra distinción, la distinción entre eterno y perpetuo, atribuyéndola a Platón. ${ }^{17}$ Boecio se refiere muy probablemente al diálogo platónico Timaios, en el que Platón define el tiempo como imagen del evo. Platón insinúa también, aunque no muy claramente, una existencia perpetua del mundo. ${ }^{18}$ Los neoplatónicos hablan de distintos grados de la eternidad. Según el Liber de causis, la causa primera está antes y encima de la eternidad; la eternidad corresponde a los espíritus puros, mientras que el alma humana está situada en el confín de la eternidad y el tiempo, participando de los dos. ${ }^{19}$ No es extraño que Tomás de Aquino, en su exposición sobre el Liber de causis, rechace esta tesis; según él, la eternidad corresponde a Dios, mientras que a los espíritus puros les corresponde un «esse perpetuum et indeficiens sine motu et temporis successione». ${ }^{20}$ Como ya se ha dicho arriba, en la exposición del Liber de causis Santo Tomás tarda un poco en aplicar a esa existencia perpe-

13 Cf.. K. E. Georges, Kleines lateinisch-deutsches Handwörterbuch, Leipzig ${ }^{5} 1885$, col. 80-81; Mittellateinisches Wörterbuch bis zum ausgehenden 13. Jahrhundert I, München 1967, col. 338-40 (aeternalis, aeternitas, aeternus), col. 34344 (aeviternitas, aeviternus, aevum, aevus). Cf. también Rhabanus Maurus, De computo, cap. 96, CCCM 44, 319: «Unde et aetas dicitur tempus quod de multis seculis instruitur, et dicta aetas quasi aevitas, id est similitudo aevi. Nam aevum est aetas perpefua, cuius neque initium neque extremum noscitur, quod Graeci vocant eonas; quod aliquando apud eos pro seculo, aliquando pro aeterno poniturr.

14 Sobre la historia del término «aevum», cf. Lexikon des Mittelalters 1, München/Zürich 1980, 193 (M. Seckler); Historisches Wörterbuch der Philosophie 1, Basel/Stuttgart 1971, pp. 88-89 (W. Wieland); Cl. Baeumker, Witelo. Ein Philosoph und Naturforscher des XIII. Jahrhunderts, BGPhMA III/2, Münster 1908, pp. 580-99; F. Beemelmans, Zeit und Ewigkeit nach Thomas von Aquino, BGPhMA XVII, Münster 1914, Heft 1; A. Maier, «Scholastische Diskussionen über die Wesensbestimmung der Zeit», en Scholastik. 26, 1951, pp. 520-56; J. Owens, «Material Substance - Temporal or Eviternal?», en New Scholasticism 56, 1982, pp. 442-61; G. A. Wilson, «Non-Being. Eviternity and Time in the Ontology of Henry of Ghent», en Mediaevalia. Textos e Estudos, pp. 77-90.

15 Boethius, Philosophiae consolatio III, metrum 9. Cf. la edición de L. Bieler en CCSL 94, p. 51. Adalboldo de Utrecht, quien compuso hacia el año 1000 un comentario al citado metrum 9 de Boecio, interpreta la palabra «aevum» como «aeternitas»; cf. R. B. C. Huygens, «Mittelalterliche Kommentare zum» «O qui perpetua». 3. «Der Kommentar des Adalbold von Utrecht», en Sacris erudiri 6, 1954, p. 410, lín. 34: «sine aevo, id est sine aeternitate».

16 Boethius, Philos. Cons. V, prosa 6,4.

17 Boethius, Philos. Cons. V, prosa 6,14: «Platonem sequentes deum quidem aeternum, mundum vero dicamus esse perpetuum.»

18. Cf. Plato, Timaios, 37 sqq. y 60 sqq. Cf. Plato, Timaeus a Calcidio translatus commentarioque instructus, ed. J. H. Waszink (= Plato latinus V). London/Leiden 1962, pp. 29-30, 46, 74, 152-155.

19 Thomas de Aquino, Expositio super Librum de causis, lectio 2, en o. c. en la nota 12, allí p. $200-201$.

20 Ibíd., o. c., 201. 
tua el nombre «aevum». Pero en su Summa theologica, redactada ya antes, dedica dos artículos enteros al problema del evo. ${ }^{21}$

La distinción entre «aeternitas» y «aevum», desconocida hasta los principios del siglo XIII, se convierte en la segunda mitad del siglo en un tema discutido entre los teólogos y filósofos con mucha intensidad. Quizás, la atención a este tema esté relacionada con las discusiones sobre la eternidad del mundo provocadas por el averroismo latino, ${ }^{22}$ quizás, fue también el interés de profundizar en el conocimiento de los ángeles (por parte de los teólogos) y de las inteligencias puras (por parte de los filósofos) lo que condujo a las especulaciones sobre la naturaleza del evo. En todo caso, muchos autores de esta época trataron sobre la distinción entre eternidad y evo/eviternidad. Mencionamos sólo, aparte de Santo Tomás, a Alexander de Hales, ${ }^{23}$ Vincentius de Beauvais, ${ }^{24}$ Witelo, ${ }^{25}$ Albertus Magnus, ${ }^{26}$ Bonaventura ${ }^{27}$ y Petrus Johannis Olivi. ${ }^{28}$

No es necesario repetir aquí las discusiones de estos maestros acerca de la distinción entre eternidad, evo y tiempo. Basta destacar tres aspectos:

El primer aspecto se refiere a la terminología. Mientras la mayoría de los autores citados utiliza la palabra «aevum», San Buenaventura y Pedro de Juan Olivi prefieren el término «aeviternitas». Buenaventura dice: «Si preguntas cómo se llama aquella medida de la duración que corresponde a las substancias espirituales, hay que responder según los santos y los filósofos: eternidad creada o evo. Pero, dado que eternidad, en el sentido estricto de la palabra, se utiliza para el increado y evo a menudo para el tiempo, es lo mejor denominar la medida de duración de las criaturas espirituales con un nombre propio, el de la eviternidad». ${ }^{29}$

Segundo: todos los autores citados tratan del evo o de la eviternidad en el contexto de la angelología. Evo y eviternidad significan la medida de duración específica de los ángeles y de los espíritus puros. Al comentar los artículos correspondientes de la Summa theologica, el cardenal Cayetano insiste en el hecho de que Santo Tomás no aplica nunca los términos evo o eviternidad a la vida de los beatos hombres y ángeles; en el contexto de la escatología, Santo Tomás habla de una participación de los hombres en la eternidad divina o de una eternidad participada. ${ }^{30}$

21 Thomas de Aquino, S. th. I q. 10 a. 5 (De differentia aevi et temporis) y a. 6 (Utrum sit unum aevum tantum).

22 Cf. R. Ramón Guerrero, Historia de la Filosofia Medieval, Madrid 1996, pp. 208-215.

23 Alexander de Hales, Summa theologica I,1, Quarracchi 1924, pp. 99-110: De aeternitate Dei. Membrum III. De comparatione aeternitatis ad aevum.

24 Vincentius de Beauvais, Speculum naturale, lib. III, cap. 74 (De differentia temporis et aevi et aeterni) y cap. 75 (De convenientia illorum trium), ed. Douai 1624, repr. Graz 1964, p. 210.

25 Witelo, «Liber de inteiligentiis», n. 55,2, en Cl. Baeumker, o.c. en nota 14, pp. 67-68; cf. el comentario de Baeumker en las pp. 583-599.

26 Albertus Magnus, Physica, lib. 4, tr. 4, cap. 4 (Digressio declarans comparationem aeternitatis ad aevum et ad tempus, in quo est de numero spatiorum) y cap. 5 (Digressio declarans, utrum idem sit nunc aeternitatis et aevi et temporis), en Albertus Magnus, Opera omnia IV,1, Münster, 1987, pp. 297-299.

27 Bonaventura, In II Sent., dist. 2, pars 1, a.l (De mensura angelicae naturae in se), q.1 (Utrum spiritualia habeant propriam naturam).

28 Petrus Johannis Olivi, Quaestiones in secundum librum sententiarum, q. 9, ed. B. Jansen, Quarracchi 1922, vol. I, pp. 159-187.

29 «Quod si quaeras, quae sit illa, respondendum secundum Sanctos et Philosophos, quod dicitur aeternitas creata aut aevum. Sed quoniam aeternitas proprie accipitur pro increato et aevum frequenter accipitur pro tempore, ideo proprio nomine potest aeviternitas appellari.» Bonaventura, In II Sent., d. 2, p.1, a.1, q. 1; ed. Quarracchi, p. 56b.

30 Cf. Thomas de Aquino, S. th. I, q. 10, a.5, en Editio leonina IV, 1888, p. 101.Cf. también Scg III, cap. 61 (Quod per visionem Dei aliquis sit particeps vitae aeternae). 
Tercero: la naturaleza del evo o de la eviternidad es determinada de distintas maneras. Vicente de Beauvais ve la peculiaridad del evo en el simple hecho de que el evo tiene principio, pero no fin, mientras que la eternidad carece de principio y de fin. A Tomás de Aquino esta declaración no le parece correcta; según él, los ángeles son esencialmente inmutables; mutación y sucesión les tocan sólo accidentalmente. ${ }^{31}$

\section{LA EVITERNIDAD DE LOS BEATOS EN LA ESCATOLOGÍA DE RAIMUNDO}

No sabemos exactamente por qué canales se enteró Lulio de la distinción entre eternidad y evo/eviternidad. ${ }^{32}$ En todo caso, se puede constatar que sigue la terminología de San Buenaventura. Como éste, Lulio prefiere la expresión eviternidad y utiliza muy pocas veces la de evo. ${ }^{33}$ Por otra parte, Lulio no sigue la doctrina de San Buenaventura acerca de la eviternidad. Buenaventura y los demás autores arriba citados tratan de la eviternidad en el contexto de la angelología, mientras que Lulio no aplica el predicado eviterno a la duración natural y esencial de los ángeles. ${ }^{34}$ Según él, los ángeles no son eviternos por naturaleza, sino que fueron creados en el tiempo y tuvieron que alcanzar la vida eviterna mediante una libre decisión facilitada por la gracia divina. ${ }^{35}$ Ya que la decisión angelical se hace en un instante inmediatamente después su creación, en una visión concreta de los ángeles coinciden fácilmente el estado de naturaleza y el de la gracia divina; pero en la especulación teórica hay que distinguirlos. Por eso, según Lulio, la vida eviternal de los ángeles no es su vida natural, sino la vida de la última perfección que viven en el paraíso o en el infierno.

Eviternidad es, pues, según Lulio un concepto escatológico que significa la medida de duración de los beatos (o condenados) ángeles y hombres.

Preguntámonos, pues, cómo Lulio concibe la eviternidad. Una primera característica es su puesto medio entre la eternidad y el tiempo. «La cabeza de la eviternidad está en el tiempo y su remate en la eternidad». Así lo dice Raimundo en uno de los proverbios del árbol ejemplifical que

31 Thomas de Aquino, S. th. I q. 10 a. 5. Cf. G. Wieland, «Kontinuum und Engelzeit bei Thomas von Aquino», en Einheit und Vielheit. Festschrift für Carl Friedrich von Weizsäcker zum 60. Geburtstag. Herausgegeben von E. Scheibe und G. Süßmann, Göttingen, 1973, pp. 77-90. El tiempo angelical se refiere según Wieland a la mutabilidad accidental de los ángeles; véase p. 89 , nota 18.

32 Cf. E. W. Platzeck, Raimund Lull, sein Leben, seine Werke, die Grundlagen seines Denkens (Prinzipientehre) 1, Roma-Düsseldorf 1962 , p. 294 , nota 142 y 2, 1964, p. 183*, nota 95. Platzeck opina que Lulio con su idea de la eviternidad depende del franciscano Petrus Johannis Olivi; los dos podrían haberse conocido en Montpellier en los años 1289/90. Pero no se puede probar un contacto entre ellos; además, sus ideas acerca de la eviternidad no coinciden.

33 La expresión «aevum aeternum» se encuentra en el Ars generalis ultima 9,338 (CCCM 75 = ROL 14, p. 197) y Ars compendiosa Dei 30, I (CCCM $39=$ ROL 13, lin. 2792).

34 Cf. Carles Llinàs i Puente, Ars angélica. L'angelologia de Ramon Llull en el context de les seves doctrines filosòfico-teológiques. Barcelona, Universidad Autónoma, Facultat de Lletres, Tesis doctoral 1995 (director: Dr. Eusebi Colomer).

35 Arbor scientiae, o.c. en nota 2: arbor aeviternalis V, 2: «Deus creavit sanctum Michaelem, et ipsum creavit in tempore [...] Et quia sanctus Michael creatus fuit in tempore, et in illo tempore se libere convertit ad recolendum [...] Deum [...] fuerunt ei qualitates appropriatae in aeviternitate $[\ldots .]$. 
se refieren al tronco del árbol eviternal. ${ }^{36}$ Otro proverbio es: «la eviternidad es espejo de la eternidad». ${ }^{37}$ Lulio reasume la sentencia platónica «el tiempo es imagen de la eternidad», ${ }^{38}$ y la diferencia y complementa, como ya lo hiciera Vicente de Beauvais: «el evo es imagen de la eternidad, el tiempo imagen del evo". ${ }^{39}$

Respecto al tiempo, la función mediadora de la eviternidad consiste en recoger y conservar los méritos adquiridos por los hombres (y ángeles) durante su vida temporal. Las buenas o malas obras no se pierden en la muerte. En su obra magna Arbor scientiae, Lulio compara los méritos y deméritos a las raíces del árbol eviternal. En la muerte, los méritos se recogen como los frutos en la cosecha ${ }^{40}$ y obtienen un valor que no se desvanece. La duración eviterna está simbolizada por el tronco del árbol. La eviternidad llega casi a la eternidad. «Tanto durará la eviternidad cuanto la eternidad. La eternidad y la eviternidad contrajeron e hicieron compañía.» Estos son dos proverbios más del árbol ejemplifical. ${ }^{41}$ Sin embargo, entre eternidad y eviternidad queda una diferencia esencial. La eviternidad es una criatura, aunque la mayor; «ninguna criatura puede ser mayor que la eviternidad ${ }^{42}$ Siguiendo a Alejandro de Hales y Vicente de Beauvais, Lulio aclara la diferencia entre eternidad y eviternidad, afirmando que la eviternidad tiene principio, aunque, de otra parte, a diferencia del tiempo, la eviternidad no tiene fin y tampoco medio. La ausencia de medio la expone Raimundo en varios proverbios: «La eviternidad no tiene medio. El movimiento no está extendido en la eviternidad. No se multiplica número en la eviternidad. Ningún ente puede exceder ni salir de la eviternidad. $»^{43}$ La ausencia de fin no significa una simple continuación indeterminada de la vida temporal. Lulio sabe muy bien que el hombre no puede obrar después de su muerte. ${ }^{44}$ En este sentido, la muerte pone un punto final a la vida humana. De esto deduce Raimundo que las obras humanas son eviternizadas en el punto final de la muerte. Lulio compara la muerte y el juicio con la cosecha en la que se recogen los frutos maduros. En lugar de la imagen del punto final, Lulio utiliza también la imagen de una línea eviternal que tiene principio en el fin de la muerte, ${ }^{45}$ pero no tiene término en ese fin. «En la eviternidad no hay término en el fin», dice el primer proverbio luliano. ${ }^{46}$

36 Es el proverbio 2 de diez proverbios. Cf. la edición del Arbol de la ciencia de Bruselas 1663, citada en nota 2, p. 364.

37 Proverbio 7.

38 Cf. nota 18.

39 Cf. nota 24.

40 Arbor scientiae (o.c. en nota 2), arbor aeviternalis 2: «Et in hac aeviternitate durant merita et culpae, quoniam in illo fine colliguntur omnia opera, quae homines fecerunt in hac vita atque quae posita sunt in una linea aeviternali, quae habet principium in fine mortis,et non potest habere finem privationis, quia est in fine iudicii.» Cf. también n. 7: «Et isti fructus ita sunt in hac vita seminati, sicut fructus plantarum, qui in hieme seminantur, ut in aestate ex eis alius fructus colligatur et multiplicetur. Quae collectio et multiplicatio significant collectionem et multiplicationem fructuum aeviternorum.»

41 Proverbios 9 y 10.

42 Proverbio 8.

43 Proverbios 3-6.

44 Arbor scientiae (o.c. en nota 2), arbor aeviternalis, 2: «In hac vita sunt opera hominum [...] et post finem non possunt esse, quoniam finis non esset hoc quod est. Sicut Martinus, qui non foret hoc quod est sine capite. Remanent ergo opera in fine aeviternitata, ita quod non sunt post finem. Et in hoc puncto finis, videlicet qui est in morte extensus, veniunt illa quae sunt aeviternitata, sicut fructus arborum, qui veniunt maturi in aestate.»

45 Cf. n. 40.

46 Proverbio 1. 
Esta idea de la eviternidad de los beatos la desarrolla Lulio bajo varios aspectos, orientándose siempre por el símbolo del árbol. Las ramas y ramos del árbol le inspiran a hablar de la división del árbol en dos partes, el paraíso y el infierno. En la edición del Arbor scientiae, publicada en Lyon en $1637,{ }^{47}$ se ve al principio del árbol eviternal un dibujo curioso: Dios padre divide mediante un hacha el árbol en dos partes. Sin duda, la afirmación de un infierno poblado por mucha gente, que Lulio comparte con la gran mayoría de los teólogos medievales, constituía un problema grave. Se plantea la cuestión: ¿cómo puede vivir y crecer un árbol dividido de tal manera? Pero dejamos aparte este problema, constatando aquí únicamente que, según Lulio, tanto el paraíso como el infierno son eviternos.

Las hojas del árbol significan las determinaciones accidentales de la vida eviterna. Por ejemplo, al considerar el «accidens» del sitio, Raimundo sitúa el paraíso en el cielo empíreo lleno de luz, mientras que el infierno está localizado en el abismo más oscuro de la tierra.

Las flores indican el fenómeno de que los actos y virtudes fundamentales de los beatos, que son, según Raimundo, los actos «bonificare, magnificare, durare, possificare, intelligere, amare, virtuificare, verificare», se abren como una flor y manifiestan mutuamente su gloria. Por otra parte, en el infierno florecen las flores de lo malo y manifiestan la perversión de las virtudes, que consiste en desbonificar, desamar etc.

El crecimiento del árbol llega a su plenitud en los frutos que significan la última perfección de los actos morales del hombre. Esta perfección tiene, según Lulio, un carácter absolutamente dinámico. No hay nada peor que la cesación de la actividad, la ociosidad y la vacuidad. En consecuencia, la perfección eviterna de la bondad consiste en el acto concertado de los tres correlativos «bonificativum, bonificabile y bonificare». Además, la recogida de los frutos del árbol eviternal no es un acto que termina en la posesión quieta y deleitosa de ellos, sino que el acto mismo de la recogida constituye el deleite de los beatos.

Por fin, nos queda a tratar un áspecto de la eviternidad hasta ahora no tocado: el aspecto cristológico. En el Arbor scientiae Raimundo Lulio utiliza casi exclusivamente argumentos de filosofía y de teología natural para aclarar la eviternización de los méritos humanos. Sólo en casos contados habla de la gracia divina, a veces del juicio final, pero nunca de Jesucristo. Con anterioridad, en el árbol apostolical, habla de Jesucristo quien, como juez, premia a los buenos y castiga a los malos, ${ }^{48}$ y después sigue un árbol dedicado únicamente a Jesucristo, el árbol divino-humanal. Sin embargo, Lulio no combina los diversos aspectos y trata de la eviternidad sin relacionarla con la encarnación del Verbo. Esto sucede sólo en los escritos posteriores.

En el Liber de syllogismis contrariis (Paris 1311$)^{49}$ Lulio destaca que no hay proporción entre la vida eviterna y el tiempo. No obstante, el hombre puede alcanzar una vida eterna porque Dios

47 Cf. nota 2.

48 Arbor scientiae (o.c. en nota 2), arbor apostolicalis. Al aclarar los artículos de la fe, Lulio dice: «Ascendit Iesus Christus ad coelos ut gloriam daret sanctis [...] Oportet ergo quod Dominus noster lesus Christus sit iudex, cum ipse sit finis, inquantum Deus et homo, omnium entium creatorum.»

49 Liber de syllogismis contrariis, n. 31 (CCCM 32 = ROL 7, p. 188): «31. Impossibile est hominem habere vitam aeternam. Ait Raimundus: Quod secundum possibile humanum sive naturam humanam et secundum virtutes cardinales impossibile est, quod homo habet vitam aeternam, cum inter vitam aeviternam et tempus nulla sit proportio. Verumtamen non est impossibile quoad possibilitatem priorum syllogismonum hominem habere vitam aeternam, cum talis vita sit quoad bene esse, magnum esse etc. Et hoc patet per hoc, quia Deus existit et agit optime, maxime etc.» Como se ve, Lulio no distingue aquí claramente entre eternidad y eviternidad. 
actúa «optime» y «maxime».Lo que significa esto lo expone Raimundo con más claridad en el Liber de quaestione valde alta et profunda (Paris 1311). ${ }^{50} \mathrm{La}$ bondad máxima de Dios exige que éste se comunique plenamente en su Hijo al mundo para superar de este modo la distancia infinita entre si y el universo. La creación de un ser destinado a la vida eviterna se hace posible por la encarnación del Hijo divino.

Estas consideraciones constituyen, sin duda, una profundización notable de la idea de la eviternidad. Por una parte, Lulio subraya la distancia infinita y la desproporción entre Dios y el mundo, entre la eternidad divina y la caducidad y vanidad de las criaturas. Por otra parte, la idea de la encarnación del Verbo divino le permite concebir un mundo creado para un fin eviterno.

\section{ACTUALIDAD DE LA IDEA LULIANA ACERCA DE LA EVITERNIDAD}

Puede parecer que hoy día la idea de la eviternidad debiera tener sólo un valor histórico. De hecho, una vez acabada la escolástica medieval y barroca, esta idea desapareció de las discusiones filosófico-teológicas. Por ello, sorprende más el hecho de que el exegeta Gerhard Lohfink, de la facultad de teología de là universidad de Tubinga, pretenda renovar esta idea medieval. ${ }^{51}$ Su punto de partida son las palabras de Jesús sobre la parusía inminente y el fin del mundo. En conformidad con la así llamada teología de la desmitificación (Rudolf Bultmann), procura eliminar todas las imaginaciones locales y temporales acerca del fin del mundo y del más allá. Pero, ¿cómo pensar entonces la vida eterna prometida a los fieles? Los representantes de la «teología dialéctica» (Karl Barth) y otros teólogos protestantes de la primera mitad de nuestro siglo (entre ellos Paul Althaus y Emil Brunner) intentaban solucionar el problema mediante reflexiones acerca de la relación tiempo-eternidad. ${ }^{52}$ No hay proporción entre la eternidad divina y el tiempo. Por consiguiente, el último día no puede venir en un punto determinado de la historia; está, más bien, inminente o presente en cada momento del tiempo. La entrada del hombre en la vida eterna no es, pues, sino el tránsito de este mundo temporal a la eternidad de Dios, tránsito que se realiza en cada momento cuando el hombre se entrega a Dios por la fe y que se hace definitivo en la muerte. En líneas generales, Lohfink adopta esa concepción de la vida eterna. Le queda sólo un

50 Liber de quaestione valde alta et profunda, dist. 2, n. 5 (CCCM $34=$ ROL 8, p. 167, lín. 590-94): «Si non fuisset Deus incarnatus, non fuisset finis aeviternatus creatus. Ratio huius est, quia inter Deum et universum est infinita distantia; sed ratione incarnationis ipsum Verbum incarnatum est medium, eo quia est Deus et homo, ut sit vita beata aeterna.»

51 Lohfink, G., «Das aevum als Denkanstoß», en G. Greshake - G. Lohfink, Naherwartung, Auferstehung, Unsterblichkeit, Freiburg i.Br., 1975, pp. 64 sqq.

52 Cf. I. Escribano-Alberca, Eschatologie. Von der Aufklärung bis zur Gegenwart, Handbuch der Dogmengeschichte IV, 7d, Freiburg. i.Br. 1987, pp. 153-161; Schmidt, H. W., Zeit und Ewigkeit. Die letzten Voraussetzungen der dialektischen Theölogie, Gütersloh 1926; Fastenrath, E., In vitam aeternam!. Grundzüge christlicher Eschatologie in der ersten Hälfte des 20. Jahrhunderts, St. Ottilien 1982, 469-508; Seven, F., Die Ewigkeit Gottes und die Zeitlichkeit des Menschen. Eine Untersüchung der hermeneutischen Funktion der Zeit in Karl Barths Theologie der Krisis und im Seinsdenken Martin Heideggers, Göttingen, 1979; Manzke, K. H., Ewigkeit und Zeitlichkeit. Aspekte fuir eine theologische Deutung der Zeit, Göttingen, 1992. 
problema: ¿cómo se puede evitar que en este tránsito del hombre a la eternidad desaparezca la figura individual del hombre, formada por su historia personal, sus experiencias, sus decisiones, sus relaciones sociales etc., y se resuelva en el mar de la eternidad divina? Lohfink ve la solución del problema en la idea medieval del evo. «El concepto medieval del evo es un concepto sumamente necesario y extremamente útil, porque libera la escatología de la alternativa unilateral tiempo-eternidad». ${ }^{53} \mathrm{La}$ gran ventaja de ese concepto consiste, según él, en el hecho de que el evo denomina un medio entre la eternidad divina y la vida temporal del hombre, un estado medio que participa de la eternidad divina, pero no coincide con ella. El evo, como estado medio, resume la vida temporal del hombre, reúne los momentos divididos por el flujo del tiempo, los contrae en un momento único y los conserva como vida purificada, glorificada y eternizada. Lohfink compara el evo con el tiempo de la cosecha y habla del tiempo reunido, resumido y clarificado. Insiste, además, en que el evo no puede concebirse como un estado quieto, sino como acto perpetuo.

La propuesta de Lohfink de reactivar la idea medieval del evo fue criticada severamente por el cardenal Joseph Ratzinger. ${ }^{54}$ El objeto de las críticas por parte de Ratzinger no es la tesis central de Lohfink. Ratzinger también habla de la vida eterna como cosecha de la vida temporal y comparte la idea de la reunión de los momentos dispersos de la vida temporal en un único instante; incluso la profundiza, exponiendo que en la memoria humana se reúnen los miembros dispersos del tiempo y que esa memoria se desliga de sus condiciones corporales en el punto de la muerte para ser eternizada por Dios. Lo que, realmente, provocaba el disgusto de Ratzinger, era la afirmación de Lohfink de que en el momento de la muerte llega a su perfección no sólo el hombre individual, sino simultáneamente todo el universo, porque en la vida eterna no hay sucesión temporal..$^{55}$ Dado que esta problemática no toca directamente a nuestro propósito, la dejamos aparte y volvemos a la idea del evo expuesta por Lohfink.

Lohfink se basa en sus reflexiones sobre el evo solamente en Santo Tomás, el cual, como hemos visto, explica el evo como medida de duración de los ángeles. Lohfink exige con toda razón que la teología, al adoptar la idea del evo, debe liberarla del contexto angelológico y trasplantarla a la escatología. Precisamente, esto ya lo había hecho Raimundo Lulio y que, desafortunadamente, desconoció Lohfink. El gran mérito de Raimundo Lulio consiste, a mi parecer, en el hecho de haber elaborado un concepto de eviternidad que media entre la eternidad de Dios y la vida temporal del

53 Lohfink, 0.c. en nota 37, p. 66: «Der mittelalterliche Begriff des aevum ist ein höchst notwendiger und äußerst hilfreicher Begriff, denn er führt die Eschatologie aus der einseitigen Alternative Zeit-Ewigkeit heraus.»

54 - Ratzinger, J., Eschatologie - Tod und ewiges Leben, Kleine katholische Dogmatik, Regensburg 1977, pp. 97-99, 150-157. Cf. la respuesta de Lohfink en el apéndice de la tercera edición de la obra citada en nota 37, pp. 145-151: «Zur Anwendung des aevum-Begriffs».

55 Lohfink, o.c. en nota 37, p. 72: «Er (der Mensch) erfährt im Durchschreiten des Todes nicht nur, daß sich seine ganze individuelle Geschichte vor Gott versammelt, sondern zugleich - durch tausend Fäden mit der eigenen Geschichte verknüpft - die Geschichte der Welt und aller Menschen. Man kann es auch so formulieren: Indem ein Mensch stirbt und eben dadurch die Zeit hinter sich läßt, gelangt er an einen ,Punkt', an dem die gesamte übrige Geschichte, gleichzeitig: mit ihm an ihr Ende kommt, mag sie auch, inzwischen ' in der Dimension irdischer Zeit noch unendlich weite Wegstrecken zurückgelegt haben.» 
hombre y que permite concebir una vida de los muertos en la que está resumida, recogida, reunida y. clarificada la historia personal de cada uno. ${ }^{56}$ «La cabeza de la eviternidad está en el tiempo y su remate está en la eternidad». ${ }^{57}$

\author{
Klaus Reinhardt* \\ Facultad de Teología \\ Trier (Tréveris), Alemania
}

56 La comparación de la vida eterna con la cosecha parece ser algo universal; se encuentra en San Pablo (1 Cor. 15 , 43: «Se siembra en vileza y se levanta en gloria») y, de una forma más abstracta, en Adalbodo de Utrecht, filósofo de tendencia platónica, o. c. en nota 15, p. 410 , lín. 33: «nihil enim aliud est aevum quam contractio totius temporis praesentialiter habita in conspectu omnia videntis.»

57 Cf. nota 36. «Caput aeviternitatis est in tempore et eius cauda in aeternitate.»

* Agradezco a mi colega y amigo José María Soto Rábanos (Madrid, C.S.I.C.) la amabilidad de haber revisado el texto castellano del artículo 